\title{
The Cost of Scaling a Reliable Interconnection Topology
}

\author{
Rachid Guerraoui and Alexandre Maurer ${ }^{(1)}$
}

\begin{abstract}
In distributed computing, many papers try to evaluate the message complexity of a distributed system as a function of the number of nodes $n$. But what about the cost of building the distributed system itself? Assuming that we want to reliably connect $n$ nodes, how does the total number of nodes of the network evolve with $n$ ? Addressing such a question lies at the heart of achieving scalability in cloud computing. In this paper, we give the explicit description of a distributed system of which any two of the $n$ nodes, for any $n$, remain connected (by a path of alive nodes and channels) with probability at least $\mu$, despite the very fact that (a) every other node or channel has an independent probability $\lambda$ of failing, and (b) the number of channels connected to every node is physically bounded by a constant. We show however that if we also require any two of the $n$ nodes to maintain a balanced message throughput with a constant probability, then $O\left(n \log { }^{1+\epsilon} n\right)$ additional intermediary nodes are sufficient, where $\epsilon>0$ is an arbitrarily small constant.
\end{abstract}

Index Terms—Scalability, reliability, degree, throughput, network

\section{INTRODUCTION}

$\mathrm{T}$ HE growth of modern networks seems to be exceeding Moore's Law [2]. More and more computers are getting connected in cloud computing centers handling massive data storage [6], [8]. We talk for example about 60,000 cores for the Blue Brain Project [5] and over 100,000 for the CERN data center [1]. Companies like Google and Microsoft have data centers with millions of servers [3]. Not surprisingly, the problem of how to achieve scalability and effectively connect a very large number of computers has been extensively studied (see Related Works, Section 9). In particular, a lot of attention has been devoted to maintaining a reasonable message throughput (i.e., avoid traffic congestion), even when the size of the network increases. A major difficulty that hinders such scalability is the bounded (by a physical constant) capacity of network components (computers and channels): there is a maximal number of messages per second that a channel can transmit, and a maximal number of channels that a node (computer) can connect. A closer look at existing cloud constructions reveals in fact that, strictly speaking, traffic congestion increases when the size of the network increases. This is without even accounting for failures: when the size of the network increases, the probability that several components of the network fail also increases, making it even more difficult to maintain any stable throughput.

This paper asks the question of the theoretical price of scalability. Assume that we want to reliably connect $n$ nodes while preserving a stable message throughput and a bounded degree. The cost of such a network is the total number of nodes required to build it (including the $n$ nodes

- The authors are with the École Polytechnique Fédérale de Lausanne (EPFL), Lausanne 1015, Switzerland.

E-mail: \{rachid.guerraoui, alexandre.maurer\}@epfl.ch.

Manuscript received 4 Sept. 2017; revised 20 Apr. 2018; accepted 4 June 2018. Date of publication 8 June 2018; date of current version 1 Sept. 2020.

(Corresponding author: Alexandre Maurer.)

Digital Object Identifier no. 10.1109/TDSC.2018.2845402 we want to connect). We seek to determine how this cost evolves with $n$. $^{1}$

We consider the case of random failures. A natural approach is to consider the probability that the subgraph of correct nodes remains connected. However, when a graph with a bounded degree $\Delta$ becomes very large, this probability approaches zero. Indeed, as a node has at most $\Delta$ neighbors, it has a fixed probability to be cut off from the rest of the network. Over a large number of nodes, the probability to have at least one node in this situation approaches zero. We thus consider a more relaxed criteria: the minimal probability that two nodes (which can be any nodes) remain connected. As we show in this paper, it is actually possible to make this probability arbitrarily high, regardless of the failure rate.

While other solutions (see the Related Works section) can also tolerate random failures, their guarantees collapse when the network reaches a certain size (i.e., the goal probability approaches zero). The main goal of this paper is to fix this problem, together with additional constraints (such as preserving a constant throughput). The choice to focus on random failures is motivated by the observation that, in practice, most failures happen randomly (and are not selected by a centralized malicious agent). This paper provides several upper and lower bounds for the randomized model, but many problems remain open within this model. Note that, despite random failures, the problem is deterministic and requires a deterministic solution. Thus, random graphs cannot be a solution here. The hypercube graph cannot be a solution either, as it does not have a bounded degree (the degree increases with the number of nodes). We discuss the case of expander graphs in the Related Works section.

1. Note that we do not require the graph to grow gracefully with $n$ here: the graph connecting $n+1$ nodes can be very different from the graph connecting $n$ nodes. 
In the following paragraphs, we informally explain the problems and their solutions. Formal definitions of the problems are provided in Section 3.

We proceed incrementally.

(1) We first address what we call the RBD (Reliable Bounded Degree) problem, on how to connect a set of nodes so that every pair can communicate (i.e., are connected by a path of alive nodes and channels) with probability at least $\mu$, assuming that any other node or channel has an independent probability at most $\lambda$ to crash [19], [30]. (We leave aside any throughput requirement as well as Byzantine failures in this first step.) Building a complete graph, connecting any two nodes with a channel is not a solution as the node degree (i.e., the number of channels connected to a given node) keeps increasing. In fact, the RBD problem might actually seem impossible without additional intermediary nodes between the $n$ nodes (acting as routers and not necessarily reliably connected to the rest). When $n$ increases, the diameter of the graph also increases: pairs of nodes become more distant from each other, inevitably dragging down the communication probability. Compensating for this loss of reliability by adding redundant paths between any pair of (distant) nodes is infeasible for the number of parallel paths is bounded by the maximal degree whereas the network diameter keeps increasing with $n$.

We show in this paper how to address the RBD problem (with no additional intermediate nodes). For any number of nodes $n$, we show how to build a graph of $n$ nodes that ensures arbitrarily high reliability while preserving a bounded degree. We proceed in two substeps. We first solve the Weak RBD (WRBD) problem, whose goal is to reliably connect $n$ nodes with a graph of bounded degree, by allowing to add intermediary nodes between these $n$ nodes, provided that their number is $O(n)$ (at most linear in $n$ ). We do so by defining a recursive graph that ensures a constant communication probability between any two given nodes (independently of their distance) with a bounded degree, expressing the communication probability as a convergent sequence, and then a tree-like layered graph reliably connecting $n$ nodes. We then use the solution to the WRBD problem to solve our seemingly stronger RBD problem, i.e., reliably connecting $n$ nodes without intermediary nodes (the construction works with any graph solving the WRBD problem). The idea is to combine several instances of a WRBD graph, each instance reliably connecting a smaller number of nodes, and to make their intermediary nodes disappear by merging them with other nodes.

(2) We then address the problem of message throughput. We model the exchanges of messages by continuous flows of messages. Each of the $n$ nodes needs to transmit the same flow of messages to the $n-1$ other nodes. ${ }^{2}$ Assuming a bound, independent from $n$, on (1) the maximal degree of the network and (2) the maximal flow of the network, i.e., the maximal flow of messages crossing each node and channel, we address the BDF (Bounded Degree and Flow) problem (first leaving aside the reliability requirement), which consists in finding a graph that enables to maintain the flow of

2. Here, "identical" means that any node $p$ sends the same quantity of messages to any two nodes $q$ and $r$, which does not mean that the messages sent to $q$ and $r$ are the same. messages between the $n$ nodes. Again, the constraint on the degree prevents a complete graph directly connecting each pair of $n$ nodes. Thus, some flows of messages will have to go through intermediary nodes (acting as routers). At first glance, one might consider using these intermediary nodes in a tree topology, of which the leaves would be the $n$ nodes. However, a tree network is problematic for all messages would need to cross the root node, making the maximal flow increase with $n$. In fact, we prove that solving the BDF problem requires at least $\Omega(n \log n)$ intermediary nodes. Basically, the bounded degree implies a distance $\Omega(\log n)$ between most pairs of nodes, and the resulting amount of messages has to be distributed over a minimal number of intermediary nodes, due to the bounded capacity. We then describe a graph solving the BDF problem using $O(n \log n)$ intermediary nodes, which matches the lower bound. Essentially, our solution is again multi-layered, and consists in stacking $O(\log n)$ layers of $O(n)$ nodes each, and then crossing the flow of messages between each layer so that (1) the flow of messages crossing each node remains constant and (2) the flows of messages are uniformly mixed when reaching the last layer. We merge the first and the last layer of the graph, enabling each one of the $n$ nodes to exchange messages with the $n-1$ other nodes.

(3) Finally, we combine the RBD and BDF problems and define the RBDF (Reliable Bounded Degree and Flow) problem. As for RDB, we assume that each node and channel has a given probability $\lambda$ to crash, and that each pair of nodes (among the $n$ initial nodes) must keep exchanging the same flow of messages with probability $\mu$. We also define a recursive graph that ensures reliable communication between any two nodes, at whatever distance they may be (w.r.t the parameters $\lambda$ and $\mu$ ). Then, we make a layer-by-layer product of this graph with the BDF multi-layered graph, in order to combine this reliability property with the bounded degree and flow properties. The number of intermediary nodes of the resulting graph then goes from $O(n \log n)$ to $O\left(n \log { }^{1+\epsilon} n\right)$, where $\epsilon$ is a positive constant that can be as small as wanted. In other words, the additional cost of the reliability property lies in a factor $\log ^{\epsilon} n$, where $\epsilon$ can be as small as wanted.

Interestingly, all our constructions have an optimal (logarithmic) diameter. Besides, they can all be extended to tolerate Byzantine failures (when the failed components, i.e., nodes or channels, behaves arbitrarily), assuming the failure rate $\lambda$ to be strictly smaller than 0.5 , by (1) increasing the level of redundancy (compared to the case of crash failures) and (2) adding several layers of majority votes to eliminate malicious messages.

Overall, the goal of this paper is to show that a network can scale without limitation while tolerating constraints such as random failures, bounded degree, bounded throughput, or all together. All these constraints derive from intrinsic limitations of network components: a component is not perfectly reliable (random failures); a node cannot be plugged to an unbounded number of channels (bounded degree); a channel cannot hold an unbounded throughput (bounded throughput). In other words, we show that bounded characteristics of network components do not prevent the network from being unbounded.

The problems, while simple, require complex and nontrivial graph constructions to be solved. We do not claim 
our solutions to these problems to be unique. In the related works section, we discuss alternative ways to solve these problems.

The Rest of the Paper is Organized as Follows. Section 2 presents our model and Section 3 defines the problems we address (WRBD, RBD, BDF, RBDF). Sections 4, 5, 6, and 7 present solutions to these problems and argue for their correctness. In Section 8, we prove our results in terms of cost. We present the related works in Section 9 and conclude in Section 10. In the supplemental material, which can be found on the Computer Society Digital Library at http:/ / doi. ieeecomputersociety.org/10.1109/TDSC.2018.2845402, we discuss the logarithmic diameter of our solutions, and explain how our solutions can be generalized to handle Byzantine failures.

Due to space limitations, all proofs are delegated to the supplemental material, available online.

\section{MODEL}

A graph is a tuple $G=(V, E)$ where $V$ is the set of nodes and $E$ is the set of channels, modeled as a set with repetition of pairs of nodes $\{p, q\} \subseteq V$ (we enable multiple channels between $p$ and $q$ ). The degree $\delta(v)$ of a node $v$ is the number of channels $(p, q)$ such that $p=v$ or $q=v$ (the number of channels connected to $v$ ). The maximal degree of graph $G$ is $\max _{v \in V} \delta(v)$. A path connecting two nodes $p$ and $q$ is a sequence of nodes $\left(u_{1}, \ldots, u_{m}\right)$ such that $u_{1}=p, u_{m}=q$ and $\forall i \in\{1, \ldots, m-1\}, u_{i}$ and $u_{i+1}$ are neighbors.

A component of a graph $G$ is any node or channel of $G$. Each component of $G$ can be either correct (functional) or crashed (failed). A correct path is a sequence of nodes $\left(p_{1}, \ldots\right.$, $\left.p_{m}\right)$ such that, $\forall i \in\{1, \ldots, m\}, p_{i}$ is correct, and $\forall i \in\{1$, $\ldots, m-1\}$, there exists a correct channel $\left\{p_{i}, p_{i+1}\right\}$. Two nodes $p$ and $q$ are connected if there exists a correct path $\left(p_{1}\right.$, $\left.\ldots, p_{m}\right)$ such that $p_{1}=p$ and $p_{m}=q$. We denote by $\left.\lambda \in\right] 0,1[$ and $\mu \in] 0,1[$ two arbitrary constants.

Fluid Message Flow (FMF). Let $S \subseteq V$ be any arbitrary set of $n$ nodes, with $n \geq 2$, representing the computers of the network that need to issue and exchange messages. The rest of the nodes are intermediary nodes corresponding to routers that forward the messages sent by the $n$ computers of $S$ : they do not issue messages of their own.

We consider a perfectly balanced distributed (peer-topeer) system: each of the nodes of $S$ sends the same quantity of messages to every other node. More precisely, we assume that each node $p \in S$ sends a flow of messages $F$, equally distributed between the $n-1$ other nodes of $S .{ }^{3}$ Thus, for any two nodes $p$ and $q$ of $S, p$ sends a flow of messages $F /(n-1)$ directed towards $q$. We now define the paths taken by these messages.

A weighted path is a tuple $(P, \alpha)$, where $P$ is a path and $\alpha$ is an arbitrary coefficient. A weighted path represents a continuous flow of messages between two nodes $p$ and $q$, where $P$ is the path used by the messages, and $\alpha$ is the fraction of messages directed towards $q$. For any two nodes $p$ and $q$ of $S$, the

3. We consider a continuous flow of messages, to abstract away the granularity of messages. This continuous flow of messages does not represent the network at a given instant, but rather the quantity of messages exchanged in a given time period, which is assumed to be relatively stable.

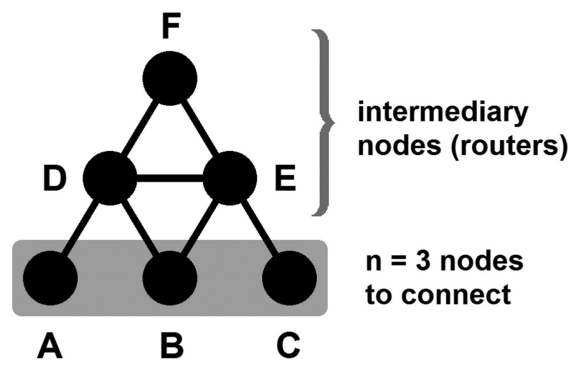

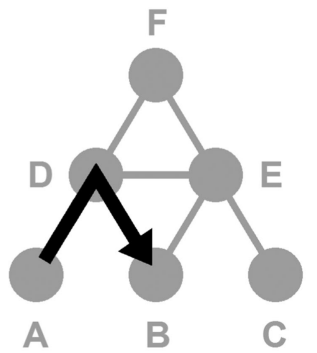

Messages from A to $B$

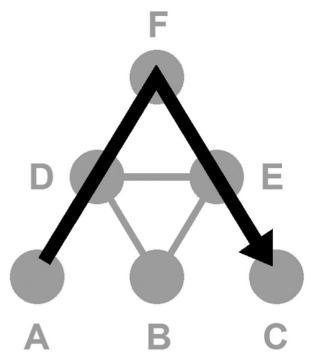

Messages from $A$ to $C$

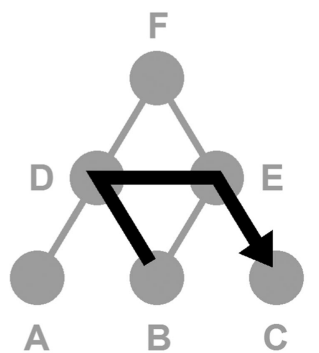

Messages from $B$ to $C$

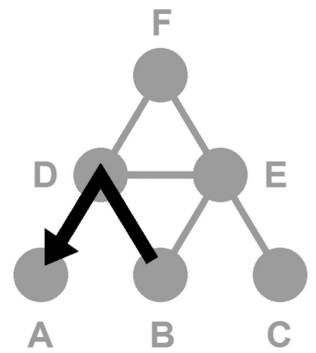

Messages from $B$ to $A$

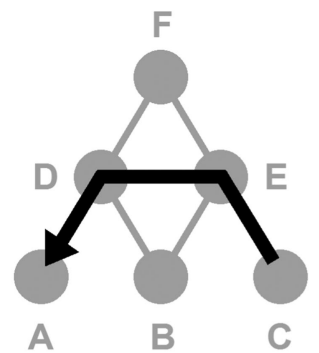

Messages from $C$ to $A$

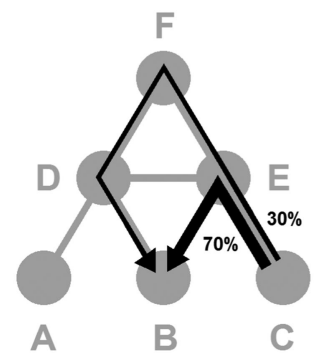

Messages from $C$ to $B$
Fig. 1. In this graph (toy example), $n=3$ nodes $A, B$ and $C$ are connected by 3 intermediary nodes $D, E$ and $F(S=\{A, B, C\}$ here). The pictures describe the (arbitrary) paths used by the flow of messages from any node to any other node. The paths are not necessarily symmetrical: for instance, the path from $A$ to $C$ and the path from $C$ to $A$ are different. Besides, the flow of messages can be split into several paths: for the messages from $C$ to $B, 70$ percent of the flow goes through $(C, E, B)$, and 30 percent of the flow goes through $(C, E, F, D, B)$. If we add up the flows of the six pictures, the maximal flow of messages is reached for node $D$.

flow of messages from $p$ to $q$ uses a set of weighted paths $R(p, q)=\left\{\left(P_{1}, \alpha_{1}\right),\left(P_{2}, \alpha_{2}\right), \ldots,\left(P_{m}, \alpha_{m}\right)\right\}$. The paths $P_{1}, P_{2}$, $\ldots, P_{m}$ are connecting $p$ to $q$, and $\alpha_{1}+\alpha_{2}++\alpha_{m}=1$. For each path $P_{i}$, the coefficient $\alpha_{i}$ corresponds to the fraction of the flow of messages using the path $P_{i}$. We illustrate this structure through a simple example in Fig. 1.

Thus, path $P_{i}$ receives a flow $\alpha_{i} F /(n-1)$ of messages from $p$ to $q$. We call the function $R$ the routing map of $S$ 
(which takes two nodes $p$ and $q$ of $S$ as input, and returns a set of weighted paths in output). For instance, in the toy example of Fig. 1, $R(C, B)=\left\{\left(P_{1}, 0.7\right),\left(P_{2}, 0.3\right)\right\}$, with $P_{1}=(C, E, B)$ and $P_{2}=(C, E, F, D, B)$.

We say that a path $\left(u_{1}, \ldots, u_{m}\right)$ crosses a node $p$ if there exists $i \in\{1, \ldots, m\}$ such that $u_{i}=p$. Similarly, we say that this path crosses a channel $\{p, q\}$ if there exists $i \in\{1, \ldots, m-$ $1\}$ such that $u_{i}=p$ and $u_{i+1}=q$. A weighted path $(P, \alpha)$ crosses a node or channel $x$ if the path $P$ crosses $x$. For a given node or channel $x$, we now define the flow of messages $f(x)$ crossing $x$. Let $\Omega=\bigcup_{\{p, q\} \subseteq S} R(p, q)$ be the set containing all weighted paths used by the nodes of $S$. Let $W=$ $\left\{\left(Q_{1}, \beta_{1}\right),\left(Q_{2}, \beta_{2}\right), \ldots,\left(Q_{k}, \beta_{k}\right)\right\}$ be the set of weighted paths of $\Omega$ crossing $x$. Then, $f(x)=\left(\beta_{1}+\beta_{2}+\ldots+\beta_{n}\right) F /(n-1)$ (the sum of the flows of messages crossing $x$ ). The maximal flow of $(G, S, R)$ is $f_{\max }=\max _{(x \in V) \vee(x \in E)} f(x)$ (the maximal flow crossing a node or channel of $G$ ).

Generalized Fluid Message Flow (GFMF). We generalize the previous model to take failures into account. Here, $R_{n}$ now takes two additional parameters $\mathcal{V}$ and $\mathcal{E}$, where $\mathcal{V}$ (resp. $\mathcal{E}$ ) represents the set of faulty nodes (resp. channels) - that is, the routing map adapts to the failures of nodes and channels in order to find correct paths, when it is possible. Thus, a set of weighted paths $R_{n}(p, q)$ becomes $R_{n}^{\mathcal{V}, \mathcal{E}}(p, q)$, and the routing map $R_{n}$ becomes $R_{n}^{\mathcal{V}, \mathcal{E}}$. If this set of paths does not contain any faulty node or channel, we say that $p$ and $q$ are reliably connected. We will first consider faults as crashes for simplicity of presentation and then, later, we will discuss Byzantine failures.

\section{Problems}

The parameters $\lambda$ (failure rate) and $\mu$ (communication probability) defined in Section 2 are fixed constants of the following problems.

The WRBD (Weak Reliable Bounded Degree) problem consists in finding, for any $n \geq 2$, a graph $G_{n}$ satisfying the three following properties:

1) Reliability. Assume each node and channel crashes with probability at most $\lambda$ (the probabilities being independent). Then, there exists a set $S_{n}$ of $n$ nodes of $G_{n}$ such that any two correct nodes of $S_{n}$ are connected with probability at least $\mu$.

2) Bounded degree. There exists a constant $\Delta$ such that, $\forall n \geq 2$, the maximal degree of $G_{n}$ is at most $\Delta$.

3) Linear number of nodes. There exists a constant $C$ such that, $\forall n \geq 2$, the number of nodes of $G_{n}$ is at most $C n$.

The RBD (Reliable Bounded Degree) problem consists in finding, for any $n \geq 2$, a graph $G_{n}$ containing exactly $n$ nodes and satisfying the two following properties:

1) Reliability. Assume each node and channel crashes with probability at most $\lambda$ (the probabilities being independent). Then, any two correct nodes of $G_{n}$ are connected with probability at least $\mu$.

2) Bounded degree. There exists a constant $\Delta$ such that, $\forall n \geq 2$, the maximal degree of $G_{n}$ is at most $\Delta$.

The BDF (Bounded Degree and Flow) problem considers the FMF model (of Section 2) and consists in finding, for any $n \geq 2$, a tuple $\left(G_{n}, S_{n}, R_{n}\right)$-where $G_{n}$ is a graph, $S_{n}$ is a set of $n$ nodes of $G_{n}$, and $R_{n}$ is a routing map of $S_{n}$-satisfying the two following properties:

1) Bounded Degree. There exists a constant $\Delta$ such that, $\forall n \geq 2$, the maximal degree of $G_{n}$ is at most $\Delta$.

2) Bounded Flow. There exists a constant $f_{0}$ such that, $\forall n \geq 2$, the maximal flow of $\left(G_{n}, S_{n}, R_{n}\right)$ is at most $f_{0}{ }^{4}$

The RBDF (Reliable Bounded Degree and Flow) problem considers the GFMF model (of Section 2) and consists in finding, for any $n \geq 2$, a tuple $\left(G_{n}, S_{n}, R_{n}^{\mathcal{V}, \mathcal{E}}\right)$-where $G_{n}=\left(V_{n}, E_{n}\right)$ is a graph, $S_{n}$ is a set of $n$ nodes of $G_{n}$, and $R_{n}^{\mathcal{V}, \mathcal{E}}$ is a routing map of $S_{n}$ - satisfying the three following properties:

1) Bounded Degree. There exists a constant $\Delta$ such that, $\forall n \geq 2$, the maximal degree of $G_{n}$ is at most $\Delta$.

2) Bounded Flow. There exists a constant $f_{0}$ such that, $\forall n \geq 2, \forall \mathcal{V} \subseteq V_{n}$ and $\forall \mathcal{E} \subseteq E_{n}$, the maximal flow of $\left(G_{n}, S_{n}, R_{n}^{\mathcal{V}, \mathcal{E}}\right)$ is at most $f_{0}$.

3) Reliability. Assume each node and channel crashes with probability at most $\lambda$ (the probabilities being independent). Let $\mathcal{V}$ (resp. $\mathcal{E}$ ) be the set of crashed nodes (resp. channels). Then, any two correct nodes of $S_{n}$ are reliably connected in $R_{n}^{\mathcal{V}, \mathcal{E}}$ with probability at least $\mu$.

\section{Solving the WRBD Problem}

In this section, we provide a solution to the WRBD problem (Section 4.1) and prove its correctness (Section 4.2).

\subsection{Solution}

We describe here a graph $G_{n}$ that solves the WRBD problem. We first give an overview, then the complete definition.

Overview. We first define the notion of layered graph, namely a graph where nodes are separated into several layers, and where only nodes of two adjacent layers can be connected. Then, we describe two layered graphs: $T_{n}$, which contains a binary tree connecting at least $n$ nodes, and $F_{n}$, which is a recursive graph defined by induction. The recursive definition of $F_{n}$ enables to preserve a constant communication probability between the first and last layer (independently of $n$ ) when $\lambda<0.01$ (Lemma 1). ${ }^{5}$ We show how to overcome this " $\lambda<0.01$ " constraint below. Besides, $F_{n}$ is defined so that the number of nodes doubles at most every 2 layers, which enables to preserve a linear number of nodes, as shown in Theorem 3. The number of layers of $T_{n}$ is adjusted so that $T_{n}$ and $F_{n}$ have the same number of layers $H_{n}$.

We consider a graph $X_{n}$, which is a layer-by-layer product of $T_{n}$ and $F_{n}$, and a graph $Y_{n}$, which puts two graphs $X_{n}$ in parallel. Doing so ensures a constant communication probability between any two nodes of the first layer.

We then apply three transformations in order to reach any communication probability $\mu$ with any failure rate $\lambda$. First, we connect several graphs $Y_{n}$ in parallel, in order to achieve any communication probability $\mu$. Second, we replicate each node, in order to simulate a failure rate $\lambda<0.01$ for each node. Third, we replicate each channel, in order to

4. The "bounded flow" constraint here represents the capacity limitations of the network.

5 . Note that this bound " $\lambda<0.01$ " is not supposed to be tight, and is simply small enough to have the desired property. 


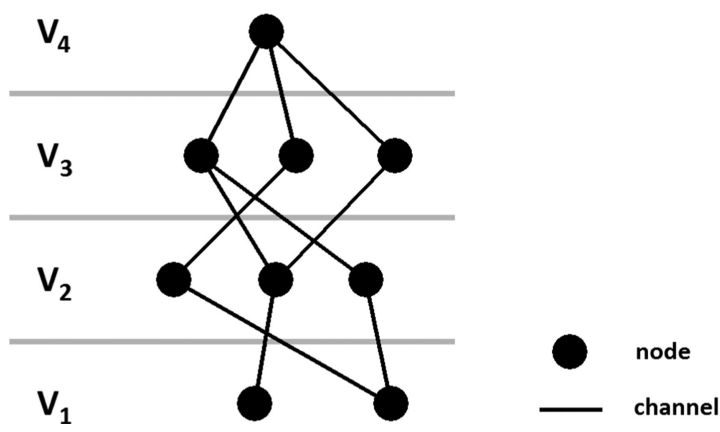

Fig. 2. A layered graph of height $H=4$.

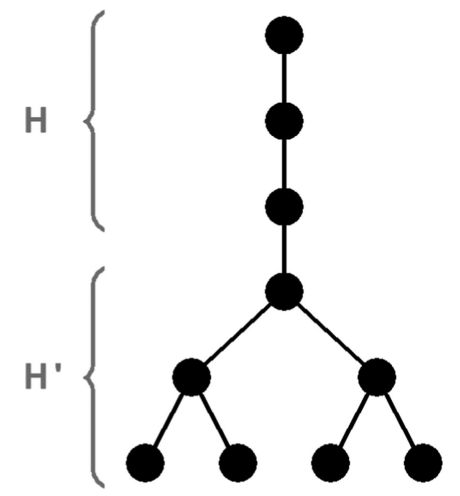

Fig. 3. Structure of graph $T_{m}$.

simulate a failure rate $\lambda<0.01$ for each channel. The graph thus obtained is $G_{n}$.

Definitions. ${ }^{6}$ We introduce a few variables below, then give a preliminary intuition of their use in the construction of the graph.

For any $n \geq 2$, let $h_{n}$ be the smallest integer such that $2^{h_{n}-1} \geq n$. Let $K_{n}$ be the smallest integer such that $2+4 K_{n} \geq h_{n}$, and let $H_{n}=2+4 K_{n}$. Let $\alpha$ be the smallest integer such that $\alpha \geq 1$ and $0.5^{\alpha} \leq 1-\mu$. Let $\beta$ be the smallest integer such that $\beta \geq 1$ and $\lambda^{\bar{\beta}} \leq 0.01$.

$h_{n}$ is the height of a binary tree connecting at least $n$ leaves. However, as our construction relies on an inductive process (see Fig. 11), The minimal height we can have is actually $H_{n}$. $\alpha$ corresponds to the number of replications of the graph, as shown in Fig. 6. $\beta$ corresponds to the number of replications of each node and channel, as shown in.

A layered graph of height $H$ is a tuple $\left(V_{1}, \ldots, V_{H}, E\right)$ satisfying the following conditions:

1) $(V, E)$ is a graph with $V=\bigcup_{i \in\{1, \ldots, H\}} V_{i}$.

2) The sets $V_{i}$ (layers) are disjoint: $\forall\{i, j\} \subseteq\{1, \ldots, H\}$, $V_{i} \cap V_{j}=\emptyset$.

3) The channels only connect neighbor layers: $\forall\{p, q\} \in$ $E$, if $p \in V_{i}$ and $q \in V_{j}$, then $|i-j|=1$.

An example of a layered graph is given in Fig. 2. By convention, in the following figures, $V_{1}$ always corresponds to the lower layer on the figure. We call $V_{1}$ the first layer and $V_{H}$ the last layer.

6. These definitions are specific to the current graph. The same goes for the definitions in the following sections.
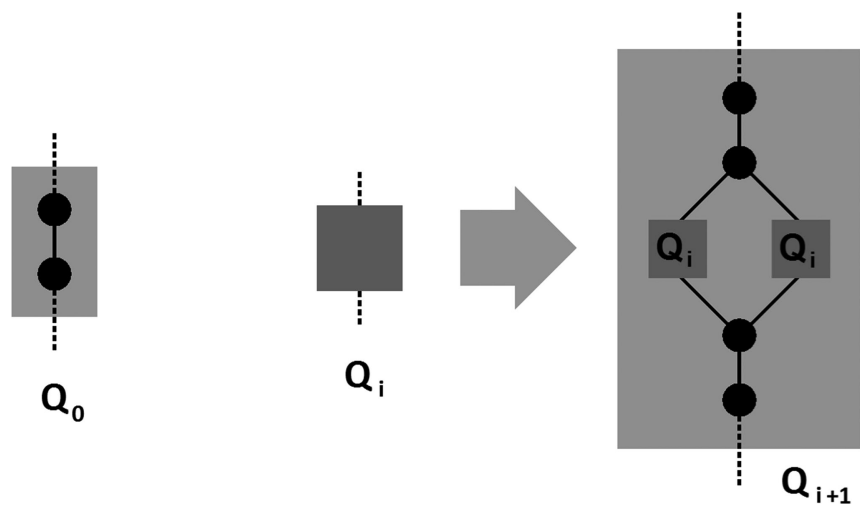

Fig. 4. Construction (by induction) of graph $Q_{i}$. The graph is defined so that the number of nodes doubles at most every 2 layers, which enables to preserve a linear number of nodes (see Theorem 3).

Graph $T_{n}$. We first define a tree-like layered graph of height $H_{n}$. Consider the layered graph represented in Fig. 3: this graph is composed of a line of height $H=3$ and of a binary tree of height $H^{\prime}=3$. In other words, $\forall i \in\left\{1, \ldots, H^{\prime}\right\}$, the layer $i$ contains $2^{i-1}$ nodes, and the $H$ remaining layers contain each 1 node. Then, $\forall n \geq 2$, we define $T_{n}$ as a similar graph with $H=H_{n}-h_{n}$ and $H^{\prime}=h_{n}$.

Graph $F_{n} . \forall k \geq 0$, we first define a layered graph $Q_{i}$ by induction. Let $Q_{0}$ be a layered graph of height 2 containing 2 nodes and 1 channel, as described in Fig. 4 . Then, $\forall i \geq 0, Q_{i+1}$ is constructed with 2 instances of $Q_{i}$ in parallel and 4 additional nodes, as described in Fig. $4\left(Q_{i+1}\right.$ has 4 more layers than $Q_{i}$ ). We now define $F_{n}$ as follows: $\forall n \geq 2, F_{n}=Q_{K_{n}}$.

Graph $X_{n} \cdot{ }^{7} \forall n \geq 2, T_{n}$ is a layered graph of height $H_{n}$, and $F_{n}$ is a layered graph of height $2+4 K_{n}=H_{n}$. As $T_{n}$ and $F_{n}$ are layered graphs, let $T_{n}=\left(V_{1}, \ldots, V_{H_{n}}, E\right)$ and $F_{n}=\left(V_{1}^{\prime}, \ldots, V_{H_{n}}^{\prime}, E^{\prime}\right)$. Then, $\forall n \geq 2$, we define the layered graph $X_{n}=\left(V_{1}^{*}, \ldots, V_{H_{n}}^{*}, E^{*}\right)$ as follows:

- $\forall i \in\left\{1, \ldots, H_{n}\right\}$, to each pair of nodes $(u, v) n V_{i} \times V_{i}^{\prime}$, we associate a unique node $p=f(u, v) \in V_{i}^{*}$ (thus, $\left.\left|V_{i}^{*}\right|=\left|V_{i}\right|\left|V_{i}^{\prime}\right|\right)$.

- $\quad$ Let $p=f(u, v)$ and $p^{\prime}=f\left(u^{\prime}, v^{\prime}\right)$. Then, $p$ and $p^{\prime}$ are neighbors in $X_{n}$ if and only if $u$ and $u^{\prime}$ (resp. $v$ and $v^{\prime}$ ) are neighbors in $T_{n}$ (resp. $F_{n}$ ).

Observe that, as the last layers of $T_{n}$ and $F_{n}$ contain 1 node, the last layer of $X_{n}$ also contains 1 node.

Graph $Y_{n} . \forall n \geq 2$, we define graph $Y_{n}$ as follows: we consider two instances of $X_{n}\left(X_{n}^{A}\right.$ and $\left.X_{n}^{B}\right)$, we merge the nodes of their first layers, and we merge the nodes of their last layers. This is illustrated in Fig. 5.

Graph $G_{n} . \forall n \geq 2$, graph $G_{n}$ is finally obtained by applying three successive transformations to $Y_{n}$ :

(1) Transformation 1 (Network replication). First, we connect $\alpha$ instances of $Y_{n}$ by merging the nodes of their first layers, as illustrated in Fig. 6 for $\alpha=3 .^{8}$

7. The definition of $X_{n}$ looks like a Cartesian product, but it is not: we do not make the product of the whole graphs, but of each pair of layer separately.

8. More precisely, let $E_{i}=\left(u_{1}^{i}, \ldots, u_{Q}^{i}\right)$ be the first layer of the $i$ th instance of $Y_{n}$ (with $i \in\{1, \ldots, \alpha\}$ ). Then, $\forall j \in\{1, \ldots, Q\}$, we merge the $\alpha$ nodes $u_{j}^{1}, \ldots, u_{j}^{\alpha}$. 


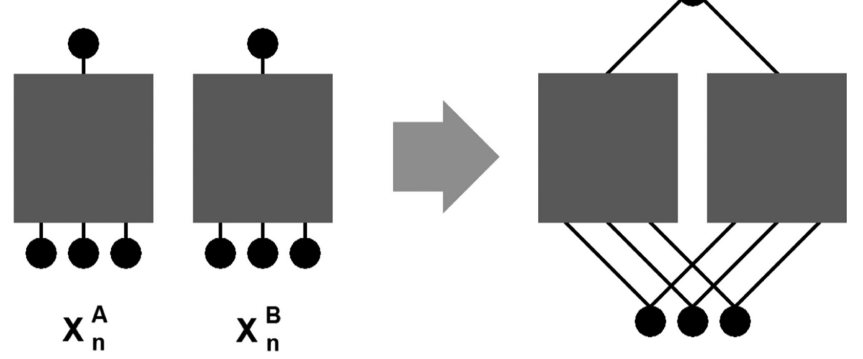

Fig. 5. Construction of graph $Y_{n}$.
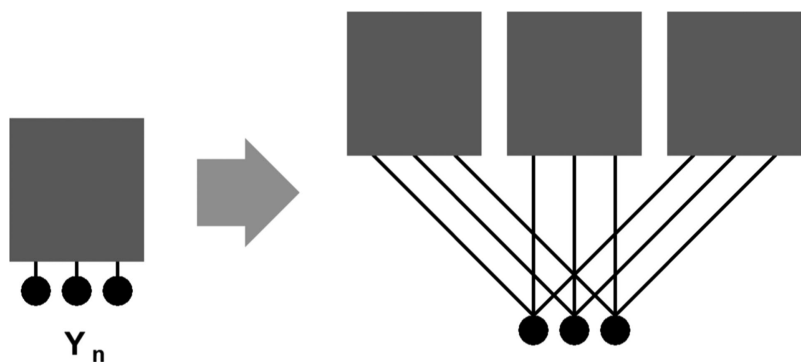

Fig. 6. Transformation 1 (Network replication) with $\alpha=3$.

(2) Transformation 2 (Node replication). Second, we replace each node $p$ by a set of $\beta$ nodes $M(p)$. Then, for each channel $\{p, q\}$, we add a channel between each node of $M(p)$ and each node of $M(q)$ (see Fig. 7a).

(3) Transformation 3 (Channel replication). Third, we replace each channel by $\beta$ channels in parallel (see Fig. 7b).

\subsection{Correctness Proof}

We prove that graph $G_{n}$ described in Section 4.1 solves the WRBD problem. For this purpose, we prove the three properties of the WRBD problem: Reliability, Bounded degree and Linear number of nodes.

In Lemma 1, we show that, for a sufficiently small failure rate $(\lambda \leq 0.01)$, the first layer and the last layer of $F_{n}$ are connected with a constant probability (independently of $n$ ). To do so, we call $P_{i}$ the probability that the first and last layer of $Q_{i}$ are connected, then express $P_{i+1}$ as a function of $P_{i}$ (according to the inductive definition of $Q_{i}$ ). Then, we show that if $P_{i} \geq 0.8$, we also have $P_{i+1} \geq 0.8$. Thus, the first and last layer of $Q_{i}$ (and thus, $F_{n}$ ) are connected with probability at least 0.8 .

In Lemma 2, we show that the first layer of $G_{n}$ contains at least $n$ nodes. Then, we consider that $S_{n}$ is a subset of the first layer of $G_{n}$ to prove the following property.

In Theorem 1, we prove the Reliability property. We first consider the case $\lambda \leq 0.01$ and $\mu \leq 0.5$ (in this case, $Y_{n}=G_{n}$ ). According to the definition of $X_{n}$ and $Y_{n}$, any two nodes of $S_{n}$ are connected to the last layer of $Y_{n}$ by two graphs $F_{n}$. Thus, the result, according to Lemma 2. We then consider that $\lambda$ and $\mu$ can have any value, and show that the 3 final transformations of Section 4.1 enable to simulate the previous situation where $\lambda \leq 0.01$ and $\mu \leq 0.5$.

In Theorem 2, we prove the Bounded degree property. As $G_{n}$ is intentionally defined as a combination of graphs with a bounded degree, the property follows.

In Theorem 3, we prove the Linear number of nodes property. We use the fact that the number of nodes of $T_{n}$ is

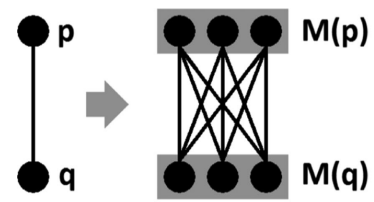

(a)

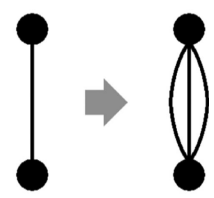

(b)
Fig. 7. Transformations 2 (Node replication) and 3 (Channel replication) with $\beta=3$.

divided by 2 every layer (starting from the first layer), while the number of nodes of $F_{n}$ at most doubles every 2 layers. Therefore, the number of nodes of $X_{n}$ (which is the combination of $T_{n}$ and $F_{n}$ ) is at least divided by 2 every 2 layers. Then, as $1+1 / 2+1 / 4+1 / 8+\leq 2$, the number of nodes of $X_{n}$ is linear in $n$, and so is the number of nodes of $G_{n}$.

Lemma 1. Assume each node and channel crashes with probability at most $\lambda$ (the probabilities being independent). If $\lambda \leq 0.01$, then $\forall n \geq 2$, the nodes of the first and last layer of $F_{n}$ are both correct and connected with probability at least 0.8 .

Lemma 2. $\forall n \geq 2$, the first layer of $G_{n}$ contains at least $n$ nodes.

Theorem 1. Assume each node and channel crashes with probability at most $\lambda$ (the probabilities being independent). Then, there exists a set $S_{n}$ of $n$ nodes of $G_{n}$ such that any two correct nodes of $S_{n}$ are connected with probability at least $\mu$.

Theorem 2. There exists a constant $\Delta$ such that, $\forall n \geq 2$, the maximal degree of $G_{n}$ is at most $\Delta$.

Theorem 3. There exists a constant $C$ such that, $\forall n \geq 2$, the number of nodes of $G_{n}$ is at most $C n$.

\section{SOLVING THE RBD PROBLEM}

In this section, we provide a solution to the RBD problem (Section 5.1) and prove its correctness (Section 5.2).

\subsection{Solution}

We describe here a graph $G_{n}{ }^{9}$ that solves the RBD problem. We first give an overview, then the complete definition.

Overview. The idea is to combine several instances of a WRBD graph, each instance reliably connecting a smaller number of nodes, and to make their intermediary nodes disappear by merging them with other nodes.

Let $W_{m}$ be any WRBD graph (for instance, the WRBD graph defined in Section 4). Then, $\forall n \geq 2$, we consider the largest integer $m$ such that the number of nodes of $W_{m}$ is at most $n$. If such a $m$ does not exist, we define $G_{n}$ as a complete graph with redundancy of channels. As it only happens for bounded values of $n$, it does not break the "Bounded degree" property.

Otherwise, we consider a set $V$ of $n$ nodes, and we split $V$ into subsets of $\lfloor m / 2\rfloor$ nodes. Then, we connect each pair of subsets with an instance of $W_{m}$ merged with the nodes of $V$. The resulting graph is $G_{n}$. Doing so ensures that any two nodes of $V$ are reliably connected. Besides, according to the "Linear number of nodes" property of $W_{m}$, the number of instances of $W_{m}$ is bounded, and so is the maximal degree of $G_{n}$.

9. The graph $G_{n}$ of each section is different, each one solving one of the four problems. 


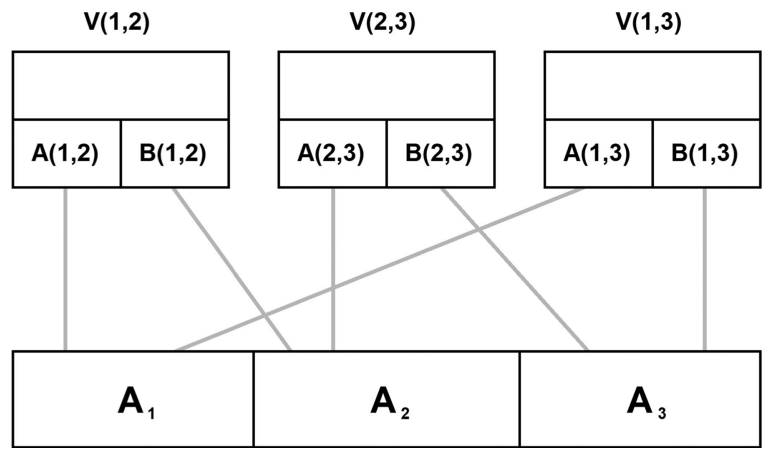

Fig. 8. Illustration of the construction of $G_{n}$ for $M=3$ (RBD problem). We represented $V(1,2), V(2,3), V(1,3)$ and $V$, as well as their subset $A(i, j), B(i, j)$ and $A_{i}$. The grey lines show how the subsets $A(i, j)$ and $B(i, j)$ are merged with the subsets $A_{i}$.

Construction of $G_{n}$. Let $n \geq 2$, and let $V$ be a set of $n$ nodes.

Let $W_{m}$ be any WRBD graph. Let $N_{m}$ be the total number of nodes of $W_{m}\left(N_{m} \geq m\right)$, and let $S_{m}$ be the set of $m$ nodes reliably connected by $W_{m}$.

If there exists no $m \geq 2$ such that $N_{m} \leq n$, then for any two nodes $p$ and $q$ of $V$, we add $\lceil\log (1-\mu) / \log (1-\lambda)\rceil$ channels between $p$ and $q$ (complete graph).

Otherwise, let $m \geq 2$ be the largest integer such that $N_{m} \leq n$. Let $M$ be the smallest integer such that $M\lfloor m / 2\rfloor \geq n$. Let $\left\{A_{1}, \ldots, A_{M}\right\}$ be any set of $M$ subsets of $V$ such that $\bigcup_{i \in\{1, \ldots, M\}} A_{i}=V$ and $\forall i \in\{1, \ldots, M\},\left|A_{i}\right|=\lfloor m / 2\rfloor$.

Then, $\forall\{i, j\} \subseteq\{1, \ldots, M\}$, we apply the following transformations. Let $W(i, j)$ be an instance of $W_{m}$, let $V(i, j)$ be the set of nodes of $W(i, j)$, and let $S(i, j)$ be the set of $m$ nodes corresponding to $S_{m}$. Let $A(i, j)$ and $B(i, j)$ be two disjoint subsets of $S(i, j)$ such that $|A(i, j)|=|B(i, j)|=\lfloor m / 2\rfloor$. We merge the $\lfloor m / 2\rfloor$ nodes of $A(i, j)$ (resp. $B(i, j)$ ) with the $\lfloor m / 2\rfloor$ nodes of $A_{i}$ (resp. $A_{j}$ ). Then, we merge the $N_{m}-2\lfloor m / 2\rfloor$ nodes of $V(i, j)-A(i, j)-B(i, j)$ with any $N_{m}-2\lfloor m / 2\rfloor$ nodes of $V-A_{i}-A_{j}$. The graph thus obtained is $G_{n}$. We illustrate this in Fig. 8.

\subsection{Correctness Proof}

We prove that graph $G_{n}$ described in Section 5.1 solves the RBD problem. For this purpose, we prove the two properties of the WRBD problem: Reliability and Bounded degree.

In Theorem 4, we prove the Reliability property. Let $p$ and $q$ be two nodes of $G_{n}$. In the case where the graph is complete, the reliability property is ensured by the number of channels between $p$ and $q$. Otherwise, it is ensured by the fact that $p$ and $q$ belong to the set $S_{m}$ of at least one instance of $W_{m}$.

In Theorem 5, we prove the Bounded degree property. We first notice that the graph is complete only when $n \leq N_{2}$. Thus, in this case, the degree is bounded. Otherwise, we show that the number of subsets of $\lfloor m / 2\rfloor$ nodes is bounded (which is a consequence of the linearity property of the WRBD problem). Thus, the number of instances of $W_{m}$ is bounded, and so is the degree of $G_{n}$.

Theorem 4. Assume each node and channel crashes with probability at most $\lambda$ (the probabilities being independent). Then, any two correct nodes of $G_{n}$ are connected with probability at least $\mu$.

Theorem 5. There exists a constant $\Delta$ such that, $\forall n \geq 2$, the maximal degree of $G_{n}$ is at most $\Delta$.
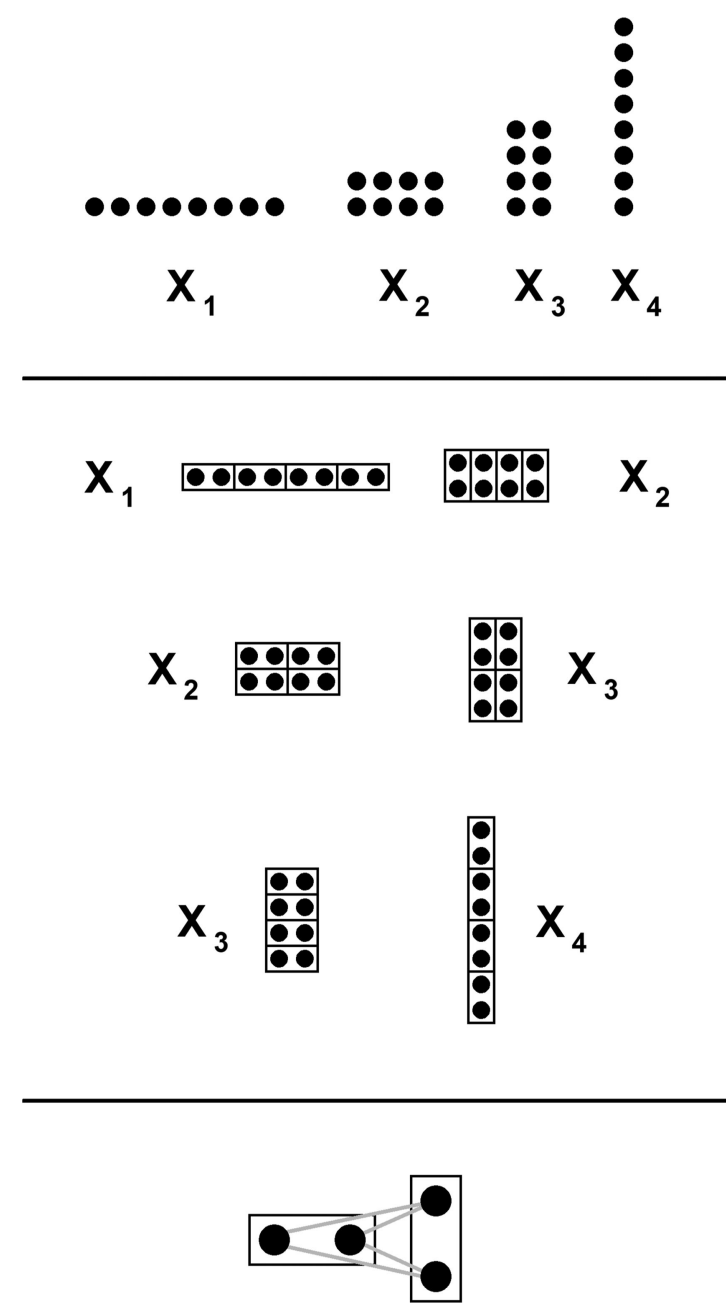

Fig. 9. Illustration of the construction of $G_{n}$ for $H=4$ (BDF problem). The first part represents the sets $X_{k}$ for $k \in\{1,2,3,4\}$. The nodes $u_{k}(i, j)$ are ordered such that $i$ (resp. $j$ ) corresponds to the horizontal (resp. vertical) axis. The second part shows how $X_{k}$ and $X_{k+1}$ are connected. $X_{k}$ (resp. $X_{k+1}$ ) is partitioned into horizontal (resp. vertical) pairs of nodes. Each pair of nodes of $X_{k}$ is connected to the pair of nodes of $X_{k+1}$ with the corresponding position, as shown in the third part.

\section{Solving the BDF Problem}

In this section, we provide a solution to the BDF problem (6.1) and prove its correctness (6.2).

\subsection{Solution}

We describe here a tuple $\left(G_{n}, S_{n}, R_{n}\right)$ that solves the BDF problem. We first give an overview, then the complete definition of $G_{n}, S_{n}$ and $R_{n}$.

Overview. To construct $G_{n}$, the intuitive idea is the following. We define a sequence $\left(X_{1}, \ldots, X_{H}\right)$ of sets of $O(n)$ nodes. $X_{1}, X_{2}, \ldots, X_{H}$ can be represented as tables of respectively $2^{H-1} \times 1,2^{H-2} \times 2, \ldots, 1 \times 2^{H-1}$ nodes (each time, the width is divided by two and the height is multiplied by two). This is illustrated in Fig. 9. Then, each node of $X_{i}$ is connected to two nodes of $X_{i+1}$ with the same height modulo 2 and the same width modulo $2^{H-i} \cdot{ }^{10}$ Finally, we

10. The demultiplexing properties of $G_{n}$ are similar to those of a butterfly [20] network. However, $G_{n}$ is defined differently. In a butterfly network, the nodes of each layer are described by an index $i$. Here, they are described by two indexes $i$ and $j\left(\right.$ " $u_{k}(i, j)$ "). 
merge $X_{1}$ and $X_{H}$ so that the sets of nodes form a cycle. As we show further, this construction enables to mix the flows of messages in a perfectly balanced way. $S_{n}$ is an arbitrary set of $n$ nodes of the first layer of $G_{n}$.

We then define the routing map $R_{n}$ as follows. The flows of messages between two nodes $p$ and $q$ of $S_{n}$ take a unique path $r(p, q)$ ( $p$ is seen as a node of $X_{1}$ and $q$ as a node of $X_{H}$ ). The path is determined by the binary decomposition of the position of $q$ in $X_{H}$ : at each new step, 0 means "go down" $\left(v_{k+1}=x\left(b_{k}\right)\right)$ and 1 means "go up" $\left(v_{k+1}=y\left(b_{k}\right)\right)$. This corresponds to the upper node and lower node in the third part of Fig. 9. We show that $r(p, q)$ actually reaches $q$ in the correctness proof.

Graph $G_{n}$. Let $H$ be the smallest integer such that $2^{H-1} \geq n$ (as $n \geq 2, H \geq 2$ ). We consider $H$ sets of nodes $\left(X_{1}, \ldots, X_{H}\right)$, containing $2^{H-1}$ nodes each. $\forall k \in\{1, \ldots, H\}$, we denote each node of $X_{k}$ by $u_{k}(i, j)$, with $i \in\left\{1, \ldots, 2^{H-k}\right\}$ and $j \in\left\{1, \ldots, 2^{k-1}\right\}$ (this is possible as $2^{H-k} \times 2^{k-1}=2^{H-1}$ ). We connect these $H$ sets of nodes with communication channels as follows. $\forall k \in\{1, \ldots, H-1\}, \forall i \in\left\{1, \ldots, 2^{H-k-1}\right\}$ and $\forall j \in$ $\left\{1, \ldots, 2^{k-1}\right\}$, let $a=u_{k}(2 i-1, j), b=u_{k}(2 i, j), x=u_{k+1}(i$, $2 j-1)$ and $y=u_{k+1}(i, 2 j)$. Then, we add the following communication channels: $\{a, x\},\{a, y\},\{b, x\}$ and $\{b, y\}$. Finally, $\forall i \in\left\{1, \ldots, 2^{H-1}\right\}$, we merge the node $u_{1}(i, 1)$ with the node $u_{H}(1, i)$. The graph thus obtained is $G_{n}$.

Set of Nodes $S_{n}$. We define $S_{n}$ as an arbitrary subset of the set $X_{1}$, containing exactly $n$ nodes. This is possible as $2^{H-1} \geq n$.

Routing Map $R_{n}$. For a given node $v \in X_{1} \cup \cup X_{H-1}$, let $k, i$ and $j$ be such that $v=u_{k}(i, j)$. Let $i_{0}$ be the smallest integer such that $2 i_{0} \geq i$. Let $x(v)=u_{k}\left(i_{0}, 2 j-1\right)$ and $y(v)=u_{k}\left(i_{0}\right.$, $2 j)$. Let $p \in X_{1}$ and $q \in X_{H}=X_{1}$. Let $j$ be such that $q=u_{H}(1$, $j)$. Let $\left(b_{1}, \ldots, b_{H-1}\right)$ be the binary sequence $(\forall k \in\{1, \ldots, H-$ $1\}, b_{k} \in\{0,1\}$ ) such that $j-1=\Sigma_{k=1}^{k=H-1} b_{k} 2^{H-k-1}$ (that is, the binary decomposition of $i-1$ ).

Let $v_{1}=p$. We define $v_{k+1}$ by induction: if $b_{k}=0$, $v_{k+1}=x\left(b_{k}\right)$, and if $b_{k}=1, v_{k+1}=y\left(b_{k}\right)$. Let $r(p, q)=\left(v_{1}\right.$, $\left.\ldots, v_{H}\right)$. Then, we define the routing map $R_{n}$ by $R_{n}(p, q)=$ $\{(r(p, q), 1)\}$.

\subsection{Correctness Proof}

We prove that the tuple $\left(G_{n}, S_{n}, R_{n}\right)$ described in Section 6.1 solves the BDF problem. For this purpose, we first prove that $R_{n}$ is actually a routing map of $S_{n}$. Then, we prove the two properties of the BDF problem: Bounded degree and Bounded flow.

In Theorem 6, we show that $R_{n}$ is a routing map of $S_{n}$. For this purpose, we show that the definition of $r(p, q)$ (with the binary decomposition of the position of $q$ in $X_{H}$ ) is so that the path actually reaches $q$. To do so, we show by induction that the $k$ first bits always reflect the position of the node crossed by $r(p, q)$ in $X_{k}$.

In Theorem 7, we prove the Bounded degree property: the degree of $G_{n}$ is at most 4 by construction.

In Theorem 8, we prove the Bounded flow property: we show that according to the definition of the routing map, each node of $X_{k}$ is crossed by $2^{k-1} \times 2^{H-k}=2^{H-1}$ paths (which is a constant). Hence, the maximal flow is bounded.

Theorem 6. $R_{n}$ is a routing map of $S_{n}$.
Theorem 7. There exists a constant $\Delta$ such that, $\forall n \geq 2$, the maximal degree of $G_{n}$ is at most $\Delta$.

Theorem 8. There exists a constant $f_{0}$ such that, $\forall n \geq 2$, the maximal flow of $\left(G_{n}, S_{n}, R_{n}\right)$ is at most $f_{0}$.

\section{Solving the RBDF Problem}

In this section, we provide a solution to the RBDF problem (Section 7.1) and prove its correctness (Section 7.2).

\subsection{Solution}

We describe here a tuple $\left(G_{n}, S_{n}, R_{n}^{\mathcal{V}, \mathcal{E}}\right)$ that solves the RBDF problem. We first give an overview, then the complete definition of $G_{n}, S_{n}$ and $R_{n}^{\mathcal{V}, \mathcal{E}}$.

Overview. Let $G_{n}^{0}$ be the BDF graph defined in Section 6.1. After introducing preliminary definitions, we first define graph $G_{n}$. For this purpose, we define 4 intermediary graphs $A_{n}, F_{n}, P_{n}$ and $X_{n}$. All these graphs are layered graphs, as introduced in Section 4.1, and have the same height $H_{n}^{\prime}$. $A_{n}$ is an variation of the previous graph $G_{n}^{0}$ with additional layers. $F_{n}$ is a recursive graph designed to satisfy the reliability property. $P_{n}$ is an adaptation of $F_{n}$ to the reliability parameters $\lambda$ and $\mu$. Similarly to Section $4.1, X_{n}$ is a layer-by-layer product of $A_{n}$ and $P_{n}$, in order to combine the properties of the previous graph $G_{n}^{0}$ with the reliability property of $P_{n}$. $G_{n}$ is finally obtained by merging the first and the last layer of $X_{n}$, similarly to $G_{n}^{0}$. $S_{n}$ is an arbitrary set of $n$ nodes of the first layer of $G_{n}$.

To define routing map $R_{n}^{\mathcal{V}, \mathcal{E}}$, the intuitive idea is the following. For any two nodes $p$ and $q$ of $S_{n}$, we first define a subgraph $W(p, q)$. Schematically, if $p^{\prime}$ and $q^{\prime}$ are the two corresponding nodes in $G_{n}^{0}$, and $r\left(p^{\prime}, q^{\prime}\right)$ is the path connecting them, then $W(p, q)$ is the instance of $B_{n}$ corresponding to $r\left(p^{\prime}, q^{\prime}\right)$ in $G_{n}$. Then, the routing map connects $p$ and $q$ with a unique path avoiding the crashed nodes and channels in $W(p, q)$ (if it exists).

Definitions. Let $\epsilon>0$ be any arbitrary positive constant. $\epsilon$ is the constant determining the cost of the graph (in terms of number of components). Therefore, it impacts many subsequent parameters.

Let $K$ be the smallest integer such that $K \geq 2^{1 / \epsilon} . K$ is a parameter involved in the definition of graph $F_{n} . \forall n \geq 2$, let $H_{n}$ be the smallest integer such that $2^{H_{n}-1} \geq n$. We define the following sequence $\left(h_{0}, h_{1}, h_{2}, \ldots\right)$ by induction: $h_{0}=1$, and $\forall i \geq 0, h_{i+1}=2+K h_{i} . \forall n \geq 2$, let $M_{n}$ be the smallest integer such that $h_{M_{n}} \geq H_{n}$. Let $H_{n}^{\prime}=h_{M_{n}}$. $H_{n}^{\prime}$ corresponds to the height of the layers graphs $A_{n}, F_{n}, B_{n}, X_{n}$ and $G_{n}$.

Let $g(x)=2 x^{K}-x^{2 K}$. Let $z$ be the smallest integer such that $g\left(\gamma_{z}\right) \geq \gamma_{z}$, with $\gamma_{z}=1-\left(1 / 2^{z}\right)$ (we show that such an integer $z$ always exists in Lemma 3 in Section 7.2), and let $\mu_{0}=\gamma_{z}$. Let $\lambda_{0}=\min \left(1-\mu_{0}, 1-\left(\mu_{0} / g\left(\mu_{0}\right)\right)^{1 /(4+2 K)}\right)$. Let $\alpha$ be the smallest integer such that $\alpha \geq 1$ and $\left(1-\mu_{0}\right)^{\alpha} \leq$ $1-\mu$. Let $\beta$ be the smallest integer such that $\beta \geq 1$ and $\lambda^{\beta} \leq \lambda_{0}$. The parameters $\alpha$ and $\beta$ impact the redundancy of nodes and channels in the definition of $B_{n}$.

Let $\left(G_{n}^{0}, S_{n}^{0}, R_{n}^{0}\right)$ be the solution to the BDF problem described in Section 6.1.

Graph $G_{n}$. To define $G_{n}=\left(V_{n}, E_{n}\right)$, we first define 4 intermediary graphs $A_{n}, F_{n}, B_{n}$ and $X_{n}$. 


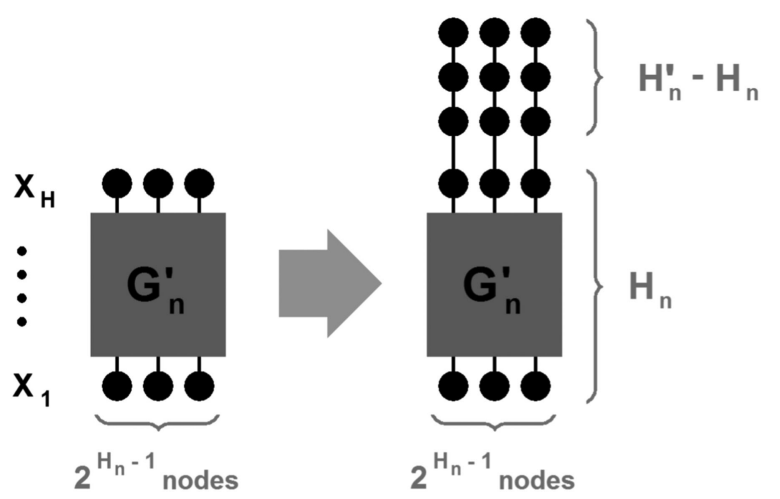

Fig. 10. Construction of graph $A_{n}$ with graph $G_{n}^{\prime}$ and $2^{H_{n}-1}$ sequences of $h_{M_{n}}-H_{n}$ nodes.

$\forall n \geq 2$, we define layered graph $A_{n}$ as follows. Consider graph $G_{n}^{0}$ and its definition in Section 6.1. The last step of the construction of $G_{n}^{0}$ consists in merging the nodes of $X_{1}$ and $X_{H}$. Let $G_{n}^{\prime}$ be graph $G_{n}^{0}$ just before this last step. Then, $G_{n}^{\prime}$ can be seen as a layered graph of height $H=H_{n}$, where the $H$ layers are $\left(X_{1}, \ldots, X_{H}\right)$. We define graph $A_{n}$ as a combination of $G_{n}^{\prime}$ and of $2^{H_{n}-1}$ sequences of $H_{n}^{\prime}-H_{n}$ nodes, such as described in Fig. 10. Thus, $A_{n}$ is a layered graph of height $H_{n}^{\prime}$.

$\forall i \geq 0$, we first define a layered graph $Q_{i}$ by induction. Let $Q_{0}$ be a layered graph of height 1 containing 1 node (see Fig. 11). Then, $\forall i \geq 0, Q_{i+1}$ is constructed with $2 K$ instances of $Q_{i}$ and 2 additional nodes, as described in Fig. 11. We now define $F_{n}$ as follows: $\forall n \geq 2, F_{n}=Q_{M_{n}}$.

$\forall n \geq 2$, graph $B_{n}$ is obtained by applying three successive transformations to $F_{n}$. Transformation 1 consists in connecting $\alpha$ instances of $F_{n}$ by merging the nodes of their first layers and then of their last layers. Transformations 2 and 3 are the same as for the WRBD graph.

$\forall n \geq 2, A_{n}$ is a layered graph of height $H_{n}^{\prime}$, and $F_{n}$ is also a layered graph of height $H_{n}^{\prime}$ (by definition of $H_{n}^{\prime}$ ). Thus, $B_{n}$ is also a layered graph of height $H_{n}^{\prime}$. As $A_{n}$ and $B_{n}$ are layered graphs, let $A_{n}=\left(V_{1}, \ldots, V_{H_{n}^{\prime}}, E\right)$ and $B_{n}=\left(V_{1}^{\prime}, \ldots, V_{H_{n}^{\prime}}^{\prime}, E^{\prime}\right)$. Then, $\forall n \geq 2$, we define the layered graph $X_{n}=\left(V_{1}^{\prime \prime}, \ldots\right.$, $\left.V_{H_{n}^{\prime}}^{\prime \prime}, E^{\prime \prime}\right)$ by the same mechanism as for the WRBD graph.

The first layer $V_{1}^{\prime \prime}$ of $X_{n}$ contains $m=2^{H_{n}-1}$ nodes, and so does its last layer $V_{H_{n}^{\prime}}^{\prime \prime}$. Let $V_{1}^{\prime \prime}=\left\{u_{1}, \ldots, u_{m}\right\}$ and $V_{H_{n}^{\prime}}^{\prime \prime}=\left\{v_{1}\right.$, $\left.\ldots, v_{m}\right\}$ (the order of numbering is unimportant here). We finally obtain graph $G_{n}$ as follows: $\forall i \in\{1, \ldots, m\}$, we merge the nodes $u_{i}$ and $v_{i}$.

Set of Nodes $S_{n}$. Let $S_{n}^{\prime}$ be any set of any $n$ nodes of the first layer of $X_{n}$ (such a set exists, as $\left|V_{1}^{\prime \prime}\right| \geq 2^{H_{n}-1} \geq n$ ). We define $S_{n}$ as the corresponding set of nodes in $G_{n}$.

Routing Map $R_{n}^{\mathcal{V}, \mathcal{E}}$. Let $p$ and $q$ be any two nodes of $S_{n}$. As $G_{n}$ is obtained by merging the nodes of $V_{1}^{\prime \prime}$ and $V_{H_{n}^{\prime}}^{\prime \prime}$ in $X_{n}$, let $p^{\prime \prime}$ (resp. $q^{\prime \prime}$ ) be the corresponding node if $V_{1}^{\prime \prime}$ (resp. $V_{H_{n}^{\prime}}^{\prime \prime}$ ).

According to the definition of $X_{n}$, let $p_{F}$ (resp. $q_{F}$ ) be the node of $A_{n}$ such that there exists a node $v$ (resp $\left.v^{\prime}\right)$ such that $p^{\prime \prime}=\pi\left(p_{F}, v\right)$ (resp. $q^{\prime \prime}=\pi\left(q_{F}, v^{\prime}\right)$ ). According to the definition of $A_{n}$, let $p_{G}$ be the node of $G_{n}^{\prime}$ corresponding to $p_{F}$, and let $q_{G}$ be the node of the last layer of $G_{n}^{\prime}$ which is connected to $q_{F}$ by a path of $H_{n}^{\prime}-H_{n}$ nodes (according to Fig. 10). Finally, let $p^{\prime}$ (resp. $q^{\prime}$ ) be the node corresponding to $p_{G}$ (resp. $q_{G}$ ) in $G_{n}^{0}$.

Let $r\left(p^{\prime}, q^{\prime}\right)$ be the path connecting $p^{\prime}$ and $q^{\prime}$ in $G_{n}^{0}$, such as defined in Section 6.1 (as shown in the proof of Theorem 6,

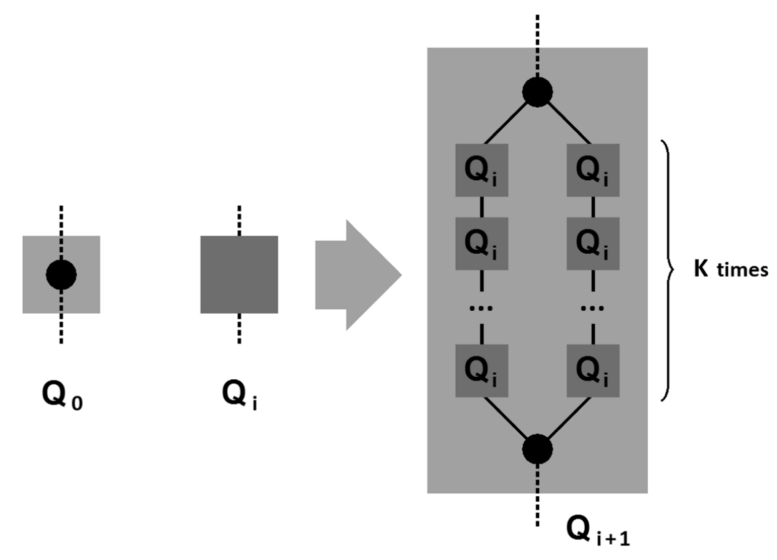

Fig. 11. Construction (by induction) of graph $Q_{i}$.

$r\left(p^{\prime}, q^{\prime}\right)$ actually connects $p^{\prime}$ and $\left.q^{\prime}\right)$. Let $r_{G}\left(p_{G}, q_{G}\right)$ be the corresponding path in $G_{n}^{\prime}$. Let $r_{F}\left(p_{F}, q_{F}\right)=\left(u_{1}, \ldots, u_{H_{n}^{\prime}}\right)$ be an extension of $r_{G}\left(p_{G}, q_{G}\right)$ connecting $p_{F}$ and $q_{F}$ in $A_{n}$ with $H_{n}^{\prime}-H_{n}$ additional nodes (see Fig. 10). $\forall i \in\left\{1, \ldots, H_{n}^{\prime}\right\}$, let $W_{i}$ be the set of nodes $w$ of $X_{n}$ such that there exists a node $v$ such that $w=\pi\left(u_{i}, v\right)$. Let $W=\bigcup_{i \in\left\{1, \ldots, H_{n}^{\prime}\right\}} W_{i}$. Let $W^{\prime}$ be the corresponding set of nodes in $G_{n}$. We define $W(p, q)$ as the subgraph containing the nodes of $W$ (and the channels connecting them) in $G_{n}$.

Now, let $\mathcal{V}($ resp. $\mathcal{E}$ ) be any arbitrary set of crashed nodes (resp. edges) of $G_{n}$. If there exists a path of correct nodes and channels connecting $p$ and $q$ in $W(p, q)$, let $\psi(\mathcal{V}, \mathcal{E}, p, q)$ be this path. Otherwise, let $\psi(\mathcal{V}, \mathcal{E}, p, q)$ be any path connecting $p$ and $q$ in $W(p, q)$. We define the routing map $R_{n}$ by $R_{n}^{\mathcal{V}, \mathcal{E}}(p, q)=$ $\{(\psi(\mathcal{V}, \mathcal{E}, p, q), 1)\}$ for any two nodes $p$ and $q$ of $S_{n}$.

\subsection{Correctness Proof}

We prove that the tuple $\left(G_{n}, S_{n}, R_{n}^{\mathcal{V}, \mathcal{E}}\right)$ described in Section 7.1 solves the RBDF problem. For this purpose, we prove the three properties of the RBDF problem: Bounded degree, Bounded flow and Reliability.

In Lemma 3, we prove a small property assumed in the description of the RBDF solution in Section 7.1.

In Theorem 9, we show the Bounded degree property, which follows from the construction of the graph.

In Theorem 10, we show the Bounded flow property: the worst case in terms of maximal flow (after merging several nodes) corresponds to our solution to the BDF problem.

In Lemma 4, we show that if the failure rate is at most $\lambda_{0}$, then the communication probability in $Q_{i}$ (and thus, in $F_{n}$ ) is at least $\mu_{0}$. This is due to the recursive definition of $Q_{i}$, which enables this property to propagate through each recursive step. In Lemma 5, we show that the three transformations between $F_{n}$ and $B_{n}$ adapt the result of Lemma 4 to any parameters $\lambda$ and $\mu$. Then, in Theorem 11, we show the Reliability property, which follows from the properties of $B_{n}$.

Lemma 3. Let $\gamma_{i}=1-\left(1 / 2^{i}\right)$. There exists an integer $i \geq 1$ such that $g\left(\gamma_{i}\right) \geq \gamma_{i}$.

Theorem 9. There exists a constant $\Delta$ such that, $\forall n \geq 2$, the maximal degree of $G_{n}$ is at most $\Delta$.

Theorem 10. There exists a constant $f_{0}$ such that, $\forall n \geq 2$, $\forall \mathcal{V} \subseteq V_{n}$ and $\forall \mathcal{E} \subseteq E_{n}$, the maximal flow of $\left(G_{n}, S_{n}, R_{n}^{\mathcal{V}, \overline{\mathcal{E}}}\right)$ is at most $f_{0}$. 
Lemma 4. $\forall i \geq 0$, let $p_{i}$ (resp. $q_{i}$ ) be the node of the first (resp. last) layer of $Q_{i}$. Suppose that $\lambda \leq \lambda_{0}$. If each node and channel crashes with probability at most $\lambda, p_{i}$ and $q_{i}$ are connected with probability at least $\mu_{0}$.

Lemma 5. $\forall n \geq 2$, let $p_{n}$ (resp. $q_{n}$ ) be any node of the first (resp. last) layer of $B_{n}$. If $p_{n}$ and $q_{n}$ are correct, and each other node and channel crashes with probability at most $\lambda$, then $p_{n}$ and $q_{n}$ are connected with probability at least $\mu$.

Theorem 11. Assume each node and channel crashes with probability at most $\lambda$ (the probabilities being independent). Let $\mathcal{V}$ (resp. $\mathcal{E}$ ) be the set of crashed nodes (resp. channels). Then, any two correct nodes of $S_{n}$ are reliably connected in $R_{n}^{\mathcal{V}, \mathcal{E}}$ with probability at least $\mu$.

\section{Cost}

In this section, we show that solving the BDF problem requires at least $\Omega(n \log n)$ nodes (8.1), then that our solution to the BDF and RBDF problems contain respectively $O(n \log n)$ and $O\left(n \log ^{1+\epsilon} n\right)$ nodes (Sections 8.2 and 8.3).

\subsection{Lower Bound on the BDF Problem}

In Theorem 12, we show that solving the BDF problem requires at least $\Omega(n \log n)$ nodes.

In broad outline, we assume a solution $\left(G_{n}, S_{n}, R_{n}\right)$ of the BDF problem. We first show that there are at least $\Omega\left(n^{2}\right)$ tuples of nodes $(p, q)$ of $S_{n}$ such that $p$ and $q$ are at distance at least $\Omega(\log n)$ from each other, due to the bounded degree. Therefore, as the flow of messages sent by each node of $S_{n}$ is divided between the $n-1$ other nodes, the sum of the flows of all nodes is $\Omega(n \log n)$. Thus, for the maximal flow to be bounded, at least $\Omega(n \log n)$ nodes are required.

Theorem 12. A graph solving the BDF problem, if it exists, contains at least $\Omega(n \log n)$ nodes.

\subsection{Cost of our BDF Solution}

In Theorem 13, we show that graph $G_{n}$ described in Section 6.1 contains $O(n \log n)$ nodes: $G_{n}$ is composed of $H$ sets $\left(X_{1}, \ldots, X_{H}\right)$ of $O(n)$ nodes each, with $H=O(\log n)$.

Theorem 13. Graph $G_{n}$, described in Section 6.1, contains $O(n \log n)$ nodes.

\subsection{Cost of our RBDF Solution}

We show that graph $G_{n}$ described in Section 7.1 contains $O\left(n \log { }^{1+\epsilon} n\right)$ nodes. In Lemma 6, we show that the layers of $F_{n}$ contain $O\left(\log ^{\epsilon} n\right)$ nodes. In Lemma 7, we show that the height of $G_{n}$ is $O(\log n)$. Then, as shown in Theorem 14, $G_{n}$ contains $O\left(\log ^{\epsilon} n\right) \times O(\log n) \times O(n)=O\left(n \log ^{1+\epsilon} n\right)$ nodes.

Lemma 6. There exists a constant $C_{1}$ such that the layers of graph $F_{n}$ contain at most $C_{1} \log { }^{\epsilon} n$ nodes each.

Lemma 7. There exists a constant $C_{2}$ such that $H_{n}^{\prime} \leq C_{2} \log n$.

Theorem 14. Graph $G_{n}$, described in 7.1, contains $O\left(n \log { }^{1+\epsilon} n\right)$ nodes.

\section{Related Works}

The area of robust network design is a vast domain. We thus focus of papers where the general objective is to build a graph with good connectivity properties.
A lot of work in distributed computing has been devoted to tolerating a specific number of failures [9], [11], [28]. A constant failure rate raises different problems when the size of the network is unbounded, e.g., even a very small failure rate can entirely change asymptotic properties.

In [15], [25], [27], random failures are considered, but the reliability criteria is that the whole graph should remain connected. In other words, for a failure rate $\lambda$ and a maximal degree $\Delta$, the probability that a single node is disconnected is at least $\lambda^{\Delta}$, and the probability that the graph remains fully connected is at most $1-\lambda^{\Delta}$. Thus, it is impossible to have an arbitrarily high reliability $\mu$ (which is required in the very definition of our problems).

In [7], [12], [26], the focus was on constructing a graph satisfying certain topological properties. In [12] and [7] however, the node degree is not bounded. In [26], the degree is bounded, and the reliability criteria is the connectivity of the graph - i.e., the number $k$ of disjoint paths between two given nodes. However, the length of these paths increases with the number of nodes. Therefore, when each node or channel has a given probability to fail, the probability that the $k$ paths are cut approaches 1 . Thus, no bound can be given on the communication probability when each node and channel has a given probability of failure.

A lot of network topologies that were proposed to reliably connect a large number of nodes with a reasonable degree [10], [13], [16], [17], [23], [24], [29], [31] were empirical and have only been experimented through simulations: their performances were evaluated only for a specific number of nodes. In [13], [23], [24], [31], traffic congestion slowly increases when the size of the network increases. In [10], [16], [17], [29], if we consider the asymptotic behavior of the proposed graphs (i.e., when the number of nodes grows), either the communication probability approaches zero, or the maximal degree approaches infinity.

For the RBD problem, our approach was to construct a specific graph (step by step) to match the desired properties. Intuitively, another idea could be to use expander graphs [14], [18], [21]. However, solving the RBD problem with expander graphs may be harder than it seems, if not impossible. We discuss this below.

We would define a graph as a $(K, A)$ vertex expander if, for any set $S$ of at most $K$ nodes, the nodes of $S$ are connected to at least $A|S|$ nodes [4]. By definition, $K$ is at most $n / A$ (where $n$ is the number of nodes).

One could then have the following intuition of proof. Let $G$ be the graph, and let $G^{\prime}$ be the graph after removing all crashed nodes and channels. Let $u$ be a node of $G^{\prime}$, and let $S_{i}$ be the $i$ th neighborhood of $u$ in $G^{\prime}$. Then, with a constant probability, we can show that $\left|S_{i+1}\right| \geq 2\left|S_{i}\right|$ (assuming that $A$ is large enough). Thus, by induction, one could deduct that $u$ is connected to a majority of the nodes of $G^{\prime}$ with a constant probability.

There is, however, at least one flaw is this reasoning.

First, to have $\left|S_{i+1}\right| \geq 2\left|S_{i}\right|$, we must have at least $\left|S_{i}\right|$ correct nodes connected to $S_{i}$ by one correct channel. These nodes represent an average fraction $(1-\lambda)^{2}$ of the neighbors of $S_{i}$ in $G$. Thus, we must have $A \geq 1 /(1-\lambda)^{2}$. As $K \leq n / A, K \leq n(1-\lambda)^{2}$.

Second, to show that $\left|S_{i+1}\right| \geq 2\left|S_{i}\right|$, we have to use the property according to which $S_{i}$ is connected to at least $A\left|S_{i}\right|$ 
nodes. However, the property only applies for $\left|S_{i}\right| \leq K$. Thus, all the sets $S_{i}$ combined contain at most $2 K+K+$ $K / 2+K / 4+\leq 4 K \leq 4 n(1-\lambda)^{2}$ nodes.

Therefore, for some values of $\lambda$ (e.g., when $(1-\lambda)^{2}$ is much smaller than $(1-\lambda) / 2)$, the sets $S_{i}$ do not cover a majority of correct nodes with a constant probability. Note that increasing $A$ does not help here.

In a nutshell, an idea could be to build a subgraph (with some reliability properties) around the initial node and the final node, until the two subgraphs intersect. However, this requires to use a property which is not satisfied for some failure rates, once the subgraph reaches a certain size. Thus, it cannot be proved that the two subgraphs intersect.

Whether or not the RBD problem can be solved using expanders graphs (or another family of graphs) remains an open problem. Such a claim would require a fully consistent proof, which does not exist to our knowledge. The contribution of this paper is to show that the RBD problem can be solved, the very nature of solution itself being secondary.

\section{Concluding Remarks}

The asymptotic behavior of a distributed system has been studied in the literature of distributed computing so far as a function of its number of nodes $n$. The parameters studied have typically been the message and memory complexities. Here, we consider, for the first time, the asymptotic reliability of the distributed system (i.e., the probability that any two nodes remain connected) and consider as a parameter the number of physical components needed to build the system. We show that it is possible to connect an arbitrarily large number of nodes with any desired level of reliability while preserving a bounded degree and a bounded throughput.

Our approach suggests several research directions. For instance, instead of considering a continuous flow of messages, we could model more accurately the granularity of messages with a probabilistic model. One could also consider the cost of physically wiring the network, and try to bound it.

\section{ACKNOWLEDGMENTS}

This work has been supported in part by the European ERC (Grant 339539 - AOC) and by the Swiss National Science Foundation (Grant 200021_169588 TARBDA).

\section{REFERENCES}

[1] CERN Computing. [Online]. Available: http://home.cern/about/ computing

[2] Microsoft: Datacenter Growth Defies Moore's Law. [Online]. Available: http:/ / www.pcworld.com/article/130921/article.html

[3] Microsoft Now has One Million Servers. [Online]. Available: http://tinyurl.com/millionservers

[4] Salil Vadhan. Expander Graphs. [Online]. Available: https:// people.seas.harvard.edu/salil/pseudorandomness/expanders. pdf

[5] The Blue Brain Project. [Online]. Available: http://bluebrain.epfl. ch/page-58110-en.html

[6] M. Armbrust, A. Fox, R. Griffith, A. D. Joseph, R. Katz, A. Konwinski, G. Lee, D. Patterson, A. Rabkin, I. Stoica, and M. Zaharia, "A view of cloud computing," Commun. ACM, vol. 53, pp. 50-58, 2010.

[7] R. Baldoni, S. Bonomi, L. Querzoni, and S. T. Piergiovanni, "Investigating the existence and the regularity of logarithmic harary graphs," Theoretical Comput. Sci., vol. 410, no. 21-23, pp. 2110-2121, 2009. doi:10.1016/j.tcs.2009.01.041.
[8] L. A. Barroso, J. Clidaras, and U. Hölzle, "The datacenter as a computer: An introduction to the design of warehouse-scale machines," in Synthesis Lectures on Computer Architecture, 2nd ed. San Rafael, CA, USA: Morgan \& Claypool, 2013.

[9] C. J. Colbourn, The Combinatorics of Network Reliability, vol. 200. New York, NY, USA: Oxford Univ. Press, 1987.

[10] P. Costa, A. Donnelly, G. O'Shea, and A. Rowstron, "CamCubeOS: A key-based network stack for 3D torus cluster topologies," in Proc. 22nd Int. Symp. High-Perform. Parallel Distrib. Comput., 2013, pp. 73-84.

[11] D. Dolev, "The Byzantine generals strike again," J. Algorithms, vol. 3, no. 1, pp. 14-30, 1982.

[12] R. Friedman, S. Manor, and K. Guo, "Scalable stability detection using logical hypercube," IEEE Trans. Parallel Distrib. Syst., vol. 13, no. 9, pp. 972-984, Sep. 2002. doi:10.1109/TPDS.2002.1036070.

[13] A. Ganguly, K. Chang, S. Deb, P. P. Pande, B. Belzer, and C. Teuscher, "Scalable hybrid wireless network-on-chip architectures for multicore systems," IEEE Trans. Comput., vol. 60, no. 10, pp. 1485-1502, Oct. 2011. doi:10.1109/TC.2010.176.

[14] D. Gillman, "A chernoff bound for random walks on expander graphs," SIAM J. Comput., vol. 27, no. 4, pp. 1203-1220, 1998.

[15] O. Goldschmidt, P. Jaillet, and R. Lasota, "On reliability of graphs with node failures," Netw., vol. 24, no. 4, pp. 251-259, 1994.

[16] A. Greenberg, J. R. Hamilton, N. Jain, S. Kandula, C. Kim, P. Lahiri, D. A. Maltz, P. Patel, and S. Sengupta, "VL2: A scalable and flexible data center network," in Proc. ACM SIGCOMM Conf. Data Commun., 2009, pp. 51-62.

[17] C. Guo, H. Wu, K. Tan, L. Shi, Y. Zhang, and S. Lu, “DCell: A scalable and fault-tolerant network structure for data centers," in Proc. ACM SIGCOMM Conf. Data Commun., 2008, pp. 75-86.

[18] S. Hoory, N. Linial, and A. Wigderson, "Expander graphs and their applications," Bulletin Amer. Math. Soc., vol. 43, no. 4, pp. 439-561, 2006.

[19] P. Jalote, Fault Tolerance in Distributed Systems. Englewood Cliffs, NJ, USA: Prentice-Hall, 1994.

[20] J. Kim, J. Balfour, and W. Dally, "Flattened butterfly topology for on-chip networks," in Proc. 40th Annu. IEEE/ACM Int. Symp. Microarchitecture, Dec. 2007, pp. 172-182. doi:10.1109/ MICRO.2007.29.

[21] J. Kleinberg and R. Rubinfeld, "Short paths in expander graphs," in Proc. 37th Annu. Symp. Foundations Comput. Sci., 1996, pp. 86-95.

[22] L. Lamport, R. E. Shostak, and M. C. Pease, "The Byzantine generals problem," ACM Trans. Program. Lang. Syst., vol. 4, no. 3, pp. 382-401, 1982.

[23] J. Li, C. Blake, D. S.J. De Couto, H. Imm Lee, and R. Morris, "Capacity of ad hoc wireless networks," in Proc. 7th Annu. Int. Conf. Mobile Comput. Netw., 2001, pp. 61-69. doi:10.1145/ 381677.381684.

[24] Y. C. Liang, Y. Zeng, E. C. Y. Peh, and A. T. Hoang, "Sensingthroughput tradeoff for cognitive radio networks," IEEE Trans. Wireless Commun., vol. 7, no. 4, pp. 1326-1337, Apr. 2008. doi:10.1109/TWC.2008.060869.

[25] S. Liu, K.-H. Cheng, and X. Liu, "Network reliability with node failures," Netw., vol. 35, no. 2, pp. 109-117, 2000.

[26] D. Loguinov, A. Kumar, V. Rai, and S. Ganesh, "Graph-theoretic analysis of structured peer-to-peer systems: Routing distances and fault resilience," in Proc. ACM SIGCOMM Conf. Appl. Technol., Architectures Protocols Comput. Commun., 2003, pp. 395-406. doi:10.1145/863955.863999.

[27] L. Mol, "On connectedness and graph polynomials," $\mathrm{PhD}$ thesis, Dalhousie Univ. Halifax, Halifax, Nova Scotia, 2016, https:// dalspace.library.dal.ca / bitstream/handle/10222/71408/ Mol-Lucas-PhD-Math-June-2016.pdf

[28] M. Nesterenko and S. Tixeuil, "Discovering network topology in the presence of Byzantine faults," IEEE Trans. Parallel Distrib. Syst., vol. 20, no. 12, pp. 1777-1789, Dec. 2009.

[29] R. Niranjan, A. Pamboris, N. Farrington, N. Huang, P. Miri, S. Radhakrishnan, V. Subramanya, and A. Vahdat, "Portland: A scalable fault-tolerant layer 2 data center network fabric," in Proc. ACM SIGCOMM Conf. Data Commun., 2009, pp. 39-50.

[30] R. D. Schlichting and F. B. Schneider, "Fail-stop processors: An approach to designing fault-tolerant computing systems," ACM Trans. Comput. Syst., vol. 1, no. 3, pp. 222-238, 1983.

[31] L. Tassiulas and A. Ephremides, "Stability properties of constrained queueing systems and scheduling policies for maximum throughput in multihop radio networks," IEEE Trans. Autom. Control, vol. 37, no. 12, pp. 1936-1948, Dec. 1992. doi:10.1109/ 9.182479 . 


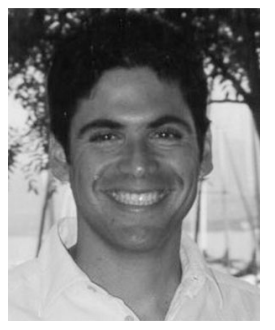

Rachid Guerraoui is a professor with the École Polytechnique Fédérale de Lausanne. He has been affiliated with the Research Center of Ecole des Mines de Paris, the Commissariat a l'Energie Atomique in Saclay, Hewlett-Packard Laboratories in Palo Alto and the Massachusetts Institute of Technology. His research is devoted to concurrent and distributed computing, from multiprocessors to wide-area networks.

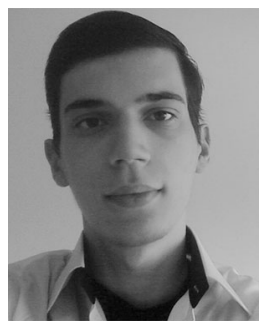

Alexandre Maurer received the graduated degree from Ecole Normale Superieure de Cachan, and the $\mathrm{PhD}$ degree in computer science from Paris 6 Sorbonne University. He is a postdoctoral researcher with the École Polytechnique Fédérale de Lausanne. His research interests include fault tolerance, safe $\mathrm{Al}$, and biological algorithms.

$\triangleright$ For more information on this or any other computing topic, please visit our Digital Library at www.computer.org/csdl. 